\title{
Hermetic Protection of Rings by Ion Beam Sputter Coating with a Broad Beam Ion Source and a W-Shaped Hollow Sputter Target
}

\author{
Wolfgang Ensinger ${ }^{1 *}$, Stefan Flege $^{1}$, Ruriko Hatada $^{1}$, Sevda Ayata $^{2}$, Takaomi Matsutani $^{1,3}$, \\ Koumei Baba ${ }^{4}$ \\ ${ }^{1}$ Darmstadt University of Technology, Department of Materials Science, 64287 Darmstadt, Germany \\ ${ }^{2}$ Dokuz Eylul University, Faculty of Science, Chemistry Department, 35160 Izmir, Turkey \\ ${ }^{3}$ Kinki University, School of Science \& Engineering, Higashiosaka 577-8502, Japan \\ ${ }^{4}$ Industrial Technology Center of Nagasaki, Omura, Nagasaki 856-0026, Japan \\ *Fax: 49-6151-16-6378, e-mail: ensinger@ca.tu-darmstadt.de
}

\begin{abstract}
Since ion-beam-based methods for materials' modification are line-of-sight techniques, it is difficult to coat three-dimensional objects completely. Here, a device is described for coating rings by ion beam sputter deposition. A broad-beam ion source is combined with a sputter target consisting of two cones, forming the shape of the letter $\mathrm{W}$ in cross-section. With this technique, steel rings were coated with thin aluminium and chromium films. Adhesion measurements at different ring positions showed that the coatings were well adhering. Electrochemical corrosion tests proved that the coating was able to protect the steel ring from aqueous corrosion.

Key words: ion beam sputter deposition, ring coating, corrosion, three-dimensional sputter target
\end{abstract}

\section{INTRODUCTION}

It is well known that ion beam based coating techniques, such as ion beam assisted deposition and ion beam sputter coating, offer several advantages such as low process temperature and very good process controllability $[1,2]$. Due to the energy input into the growing films via the kinetic energy of the ions, usually dense microstructures are obtained without the need of heating the substrates additionally. This is favourable e.g. for temperaturesensitive substrates. However, there is a basic draw-back of ion beam based methods. When a directed beam of ions is used, the process is a line-of-sight technique, i.e. only that part of a sample which is "seen" by the ion beam is treated. For this reason, the coating of three-dimensional objects is difficult. It is particularly problematic to deposit hermetic protective films on three-dimensional objects, such as rings. Often, the samples and/or the ion beam has to be manipulated which makes the process complicated. Therefore, in such cases usually plasma-based methods with their high so-called microthrowing power. i.e. coating "round the corner", are used. However, there is a possibility to apply ion beam sputtering nevertheless, when the sputter target is shaped according to the substrate to be coated. In the following, a method is described which allows to coat rings completely and thus protect them from corrosion.

\section{SET-UP OF SPUTTER-COATING APPARATUS}

The apparatus consists of a cubic vacuum chamber, equipped with a turbomolecular pump combined with a rotary fore-pump which generates a base pressure of $10^{-5}$ Pa. Fig. 1 shows a photograph. On top, a holder for substrate and sputter target is mounted. In front, a multi-aperture radiofrequency ion source with a beam aperture of $200 \mathrm{~mm}$ diameter, mounted horizontally, is visible. It operates at $13.56 \mathrm{MHz}$. The maximum acceleration voltage is $10 \mathrm{kV}$; the available current is several $\mathrm{mA}$. The source is operated with argon gas at a typical chamber gas pressure of $3 \times 10^{-3} \mathrm{~Pa}$. There is a movable shutter in front of the ion source.

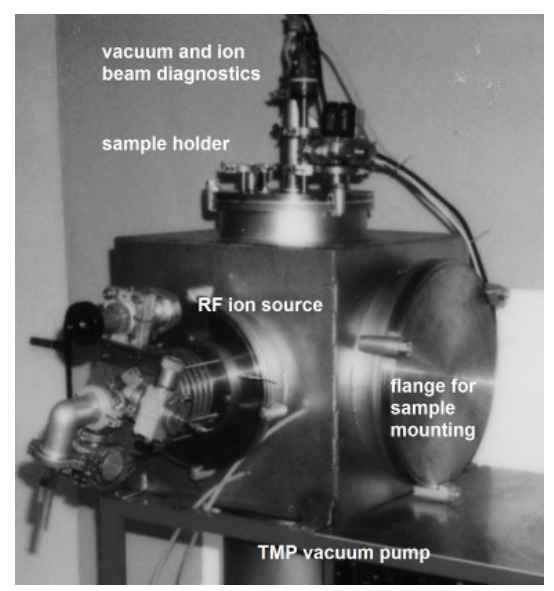

Fig. 1: Photograph of ion beam sputter coater with vacuum chamber consisting of a stainless steel cube; the ion source is mounted horizontally; sputter target and sample holder are mounted from top.

In contrast to most sputter targets, the present one is not flat, but has a three-dimensional shape. It consists of a concave cone, with a convex cone-tip inside (see schematic presentation in Fig. 2). The cone angles are $45^{\circ}$. The base of the cone tip has a diameter of $100 \mathrm{~mm}$, the outer diameter is $200 \mathrm{~mm}$. In cross-section, the sputter target has the shape of the letter $\mathrm{W}$.

In all positions, the ion beam impinges onto the sputter target at an angle of $45^{\circ}$. It releases atoms in all directions, however, with a preferential forward direction. 
The sputter target holder is made of copper. A water cooling pipe is brazed to it. Different materials can be mounted onto it. In the present case, it was $\mathrm{Al}$ and $\mathrm{Cr}$, respectively. The substrate is mounted in front of the sputter target. It is shielded from direct ion irradiation by a ring, which is a water cooled pipe. This ring is also the sample holder.

The coating process is carried out in two steps. First, the ring is coated from one side, thereafter it is turned around and then it is coated from the second side.

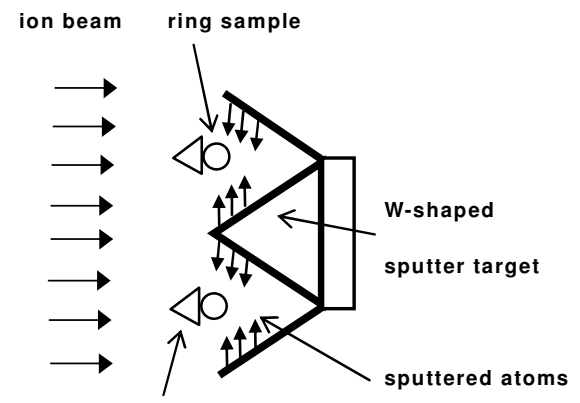

ion beam shield and sample holder

Fig. 2: Schematic presentation of process with double conical sputter target (W-shaped in cross-section) showing the direction of sputtered atoms

\section{EXPERIMENTAL DETAILS}

Steel rings with a central diameter of $100 \mathrm{~mm}$ and a thickness of $8 \mathrm{~mm}$ were mounted on the sample holder ring. A sputter target of an $\mathrm{Al}$ sheet (99.9\% purity) and another one of stainless steel AISI 304, galvanically coated with $100 \mu \mathrm{m} \mathrm{Cr}$ were used. The steel rings were coated according to the above mentioned 2-step-process. The process time was between 30 minutes and $1 \mathrm{~h}$. The coating thickness was around $0.1 \mu \mathrm{m}$ for $\mathrm{Al}$ and $0.2 \mu \mathrm{m}$ for the $\mathrm{Cr}$ coating. It was estimated from surface profiler (Dektak) measurements at edges of shielded parts.

For coating adhesion testing, rings were cut in 10 segments. Pins were glued to it with epoxy glue (cured at $120{ }^{\circ} \mathrm{C}$ for $1 \mathrm{~h}$ ) and were pulled off perpendicularly (so-called pull off or pin pull test). The pull-off force was recorded.

Elemental depth profiles of positive secondary ions were taken with secondary ion mass spectrometry (SIMS) with $2.5 \mathrm{kV} \mathrm{O}_{2}$ ions for sputtering.

Electrochemical corrosion tests (potentiodynamic current-vs-potential plots) [3] were taken in a standard three-electrode set-up with Standard Calomel Electrode (SCE) as reference electrode and a platinized Titanium plate with a diameter of $100 \mathrm{~mm}$ as counter electrode. The corrosive solution was dilute acetic acid concentration $1 \mathrm{~N}$ ), buffered with Sodium acetate to $\mathrm{pH}$ 5.6. The temperature was $25{ }^{\circ} \mathrm{C}$. The rings were contacted with a screw, which was shielded from the corrosive solution with an inert resin in order to avoid contact with it. They were completely immersed in the corrosive solution in a $1 \mathrm{~L}$ beaker. The potential scan rate was $0.01 \mathrm{~V} \mathrm{~s}^{-1}$. The system was controlled by a potentiostat which recorded the current response logarithmically.

\section{RESULTS}

3.1. Element depth profiles

Visual inspection of the coatings and examination under the light microscope gave a uniform appearance with a silver shine both of the $\mathrm{Cr}$ and the $\mathrm{Al}$ coating.

The deposition of $\mathrm{Al}$ and $\mathrm{Cr}$ was verified by taking element depth profiles by Secondary Ion Mass Spectrometry.

Fig. 3a shows the graph of the $\mathrm{Cr}$ coating. Isotopes of $\mathrm{Cr}$ in their natural abundance were found. The coating shows a broad transition to the steel substrate. Whether or not the metals were oxidized cannot be decided since according to the standard procedure for measuring depth profiles of metals, oxygen primary ions were taken. However, since the oxygen distribution shows a flat profile with depth, one can assume that the metals were at least not oxidized to a large extent.

A similar result was obtained for the aluminium coatings.

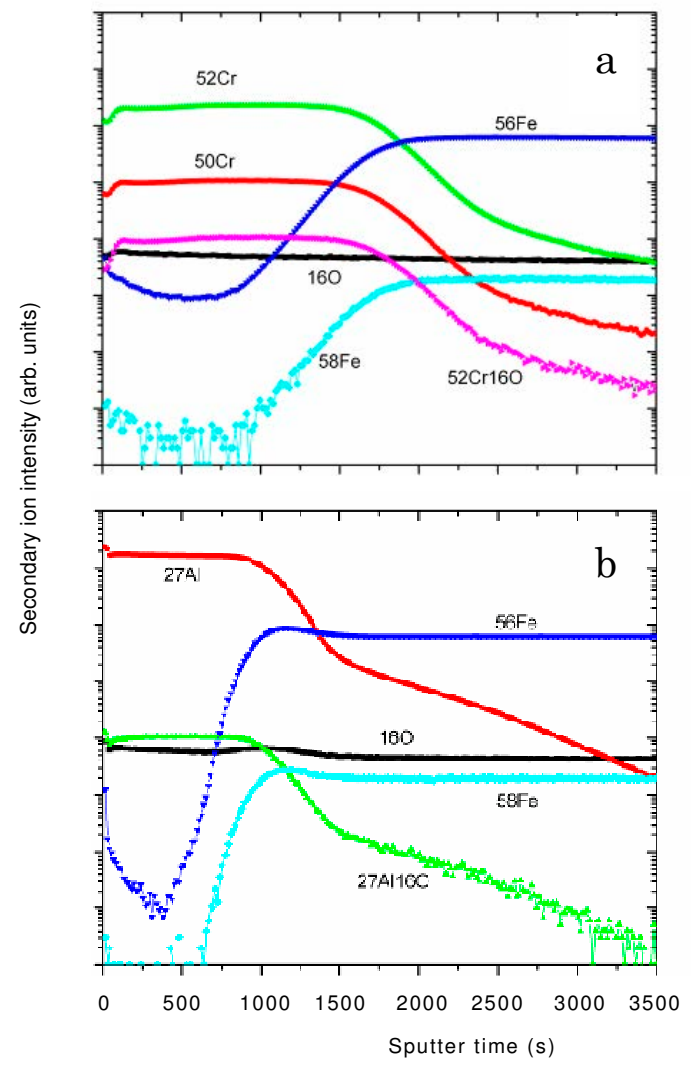

Fig. 3: SIMS element profiles of ion beam sputter coatings on steel ring substrate, a) chromium, b) aluminium

\subsection{Adhesion test results}

In order to receive a representative result on adhesion of the coatings to the substrate, the rings were cut in 10 equal segments and pin pull tests were carried out with each one. The pins were glued to the coating with high-strength epoxy glue which requires a curing temperature of $120^{\circ} \mathrm{C}$. It is assumed that this temperature does not deteriorate the coating features.

However, the cured polymer might exert some stress to the film/substrate system leading to early failure. 
The test is limited by the cohesive strength of the epoxy glue itself. This is around $80 \mathrm{MPa}$. That means that coatings where the glue itself fails might have an even higher adhesive strength.

Table I shows pull off forces of $\mathrm{Al}$ and $\mathrm{Cr}$ films. It turns out that all coatings exhibited good adhesion. Not a single one failed at low loads. Even the lowest results are beyond $60 \mathrm{MPa}$. These are similar values as obtained by sputter deposition (however, usually at higher substrate temperatures) and they are higher than those obtained by electron beam evaporation which are usually below 20 $\mathrm{MPa}$. In general, it can be stated that the deposition method gives uniformly high adherence values.

Table I: Pull off adhesion test results of $\mathrm{Al}$ and $\mathrm{Cr}$ coatings on steel ring segments:

Position on ring circumference ( $340 \mathrm{~mm}$ ); pull force $\mathbf{F}$ of adhesive failure; $\uparrow$ means failure of epoxy glue (no adhesive failure of coating)

\begin{tabular}{|c|c|c|c|c|c|c|c|c|c|c|}
\hline $\begin{array}{c}\text { Position } \\
(\mathrm{mm})\end{array}$ & 0 & 34 & 68 & 102 & 136 & 170 & 204 & 238 & 272 & 305 \\
\hline $\begin{array}{c}\text { F: Al } \\
(\mathrm{MPa})\end{array}$ & 72 & $81 \uparrow$ & 68 & $78 \uparrow$ & 77 & $82 \uparrow$ & $79 \uparrow$ & 77 & 78 & $83 \uparrow$ \\
\hline $\begin{array}{c}\text { F: Cr } \\
(\mathrm{MPa})\end{array}$ & 68 & $80 \uparrow$ & $82 \uparrow$ & 74 & 74 & $79 \uparrow$ & 72 & 73 & 69 & 75 \\
\hline
\end{tabular}

\subsection{Corrosion test results}

The samples were completely immersed in buffered acetic acid. In this medium, the steel corrodes due to anodic dissolution of iron. The cathodic counter reaction is hydrogen evolution when hydrated protons from the acid are discharged and form molecular hydrogen:

$$
\begin{gathered}
\mathrm{Fe} \rightarrow \mathrm{Fe}^{++}+2 \mathrm{e}^{-} \\
2 \mathrm{H}_{3} \mathrm{O}^{+}+2 \mathrm{e}^{-} \rightarrow \mathrm{H}_{2}+2 \mathrm{H}_{2} \mathrm{O}
\end{gathered}
$$

The corrosion can be prevented when the contact between sample and corrosive medium is prevented by coating. Materials which do not corrode in this medium can be used as protective film. Chromium and aluminium are protected by a dense natural non-corrosive oxide film. Thus, they can serve as protective coating.

In order to evaluate the corrosion performance, voltammetric measurements were carried out. The samples were immersed into the electrolyte and kept at open circuit potential for $30 \mathrm{~min}$, then the potential scan was started from there. In order to obtain a representative information on the corrosion performance, the samples were completely measured. Several rings were tested.

Fig. 4 shows the current-vs-potential plots of uncoated steel and of several Al-coated ring specimens.

The measurements show that the anodic branch of the curve of uncoated steel starts at a potential below -0.6 $\mathrm{V}_{\text {SCE }}$. Upon scanning the potential in anodic direction, a steep rise in current density is observed, representing rapid iron dissolution. At a potential around $-0.3 \mathrm{~V}$, the dissolution current density is around $10 \mathrm{~mA} \mathrm{~cm}^{-2}$.

In contrast, in case of the coated samples, the currents initially are very low. The potentials are shifted into positive direction by more than $100 \mathrm{mV}$. The currents rise only slowly, reaching several tens of $\mu \mathrm{A} \mathrm{cm}$ at a potential of $-0.3 \mathrm{~V}$.

Hence, the observed reduction in anodic iron dissolution is almost 3 orders of magnitude, corresponding to very slow corrosion. The residual current is due to dissolution of Fe through micropores, which is often observed in thin films. These results are comparable to such obtained for sputter coating and ion beam assisted deposition of flat samples [3]. This proves that the method developed is suitable for protecting rings from corrosion. Results of all samples measured were in the same order. None out of 10 samples measured showed higher currents; no outliers were observed. This means that no uncoated areas had been left over by the process and no larger pores or flaws had bee obtained, also no adherence failure had occurred during the corrosion tests. This is a hint for a very good reliability of the coating method.

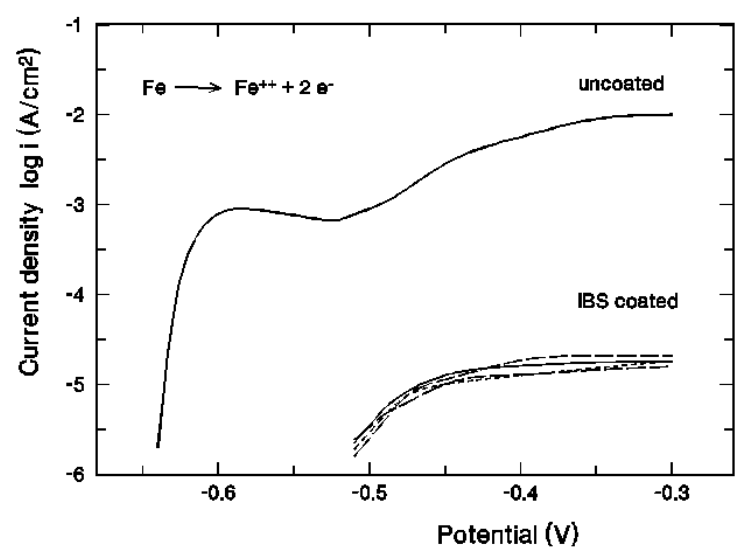

Fig. 4: Current/potential plots (anodic branch) of an uncoated steel ring and several $\mathrm{Al}$ ion beam sputter (IBS) coated rings

\section{DISCUSSION}

In contrast to deposition of protective films by e.g. electron beam evaporation, ion beam sputter deposition leads to atomic species with higher kinetic energy. In comparison, this results in favourable features such as good adhesion, high structural density, and purity. These are due to the energy of the deposited surface atoms, the so-called ad-atoms, which are able to move on the substrate surface and arrange themselves to dense structures. In contrast, in electron beam evaporation onto a substrate at room temperature, the ad-atoms can hardly move. They stay at the place of deposition, and micropores and overhanging structures may result.

In the presented set-up, the ion incidence angle is $45^{\circ}$, leading to an enhanced sputter coefficient. A part of the incident ions is reflected. However, they are not lost, since their majority hits the opposite face of the sputter target where they again increase the sputter rate. A small part of ions, impinging at the outer edge of the sputter target, may even hit the sample, leading to a further energy input and to ion beam induced effects such as ion beam mixing.

Table II shows in comparison the influence of ion energy and ion incidence angle on sputter coefficient and ion reflection; the ion energy was $10 \mathrm{keV}$. The data were calculated with SRIM using the calculation mode for sputter coefficient, based on full cascade calculation [4,5]. 
Under the present conditions with an incidence angle of $45^{\circ}$ the calculated sputter coefficient is around 6 , i.e. in average each $\mathrm{Ar}$ ion generates $6 \mathrm{Al}$ atoms to be deposited. Almost 3\% of the ions are reflected. By changing the angle of the sputter target, i.e. making the $\mathrm{W}$ more or less flat, the sputter coefficient can be varied between 3 and 11 , when ion incidence angles between $30^{\circ}$ and $60^{\circ}$, with respect to the surface normal, are chosen. Similarly, the percentage of reflected ions can be varied between $<1 \%$ and almost $10 \%$. Therefore, further studies should be focused on the shape of the sputter target. With the incidence angle via the sputter target shape, deposition rate and assisted ion irradiation can be varied. These parameters may influence the microstructure of the coating.

Table II: Sputtering coefficient and reflection yield (percentage of reflected or backscattered ions) of $10 \mathrm{keV}$ $\mathrm{Ar}$ ions, impinging on an $\mathrm{Al}$ target, for different ion incidence angles; calculated with SRIM [5]

\begin{tabular}{|c|c|c|}
\hline $\begin{array}{c}\text { Incidence } \\
\text { angle }\left[{ }^{\circ}\right]\end{array}$ & $\begin{array}{c}\text { Sputtering } \\
\text { coefficient }\end{array}$ & $\begin{array}{c}\text { Reflection } \\
\text { yield [\%] }\end{array}$ \\
\hline 30 & 3.5 & 0.6 \\
\hline 45 & 6.1 & 2.7 \\
\hline 60 & 11.5 & 9.7 \\
\hline
\end{tabular}

Another parameter is the ion acceleration voltage, defining the kinetic ion energy for a given charge state. Technically, the extraction voltage often is correlated to the ion beam density. Both together determine the power input into the sputter target and, as a consequence, the heating. Table III shows the sputtering coefficient and the reflection yield for ion energies between $5 \mathrm{keV}$ and 20 $\mathrm{keV}$. The calculation shows that the ion energy in the observed range has only a minor influence on sputtering yield and reflection yield. Therefore, it is expected that the ion source can be operated over a comparatively wide acceleration range without major influence. This has, however, to be tested with experiments.

Table III: Sputtering coefficient and reflection yield of an $\mathrm{Al}$ target with Ar ions impinging at $45^{\circ}$ for different ion energies, calculated with SRIM [5]

\begin{tabular}{|c|c|c|}
\hline $\begin{array}{c}\text { Ion Energy } \\
{[\mathrm{keV}]}\end{array}$ & $\begin{array}{c}\text { Sputtering } \\
\text { coefficient }\end{array}$ & $\begin{array}{c}\text { Reflection } \\
\text { yield [\%] }\end{array}$ \\
\hline 5 & 5.4 & 3.1 \\
\hline 10 & 6.0 & 2.7 \\
\hline 15 & 6.1 & 2.2 \\
\hline 20 & 6.3 & 1.8 \\
\hline
\end{tabular}

Apart from ion beam parameters, further studies may deal with sputter targets consisting of two or more elements, giving alloy coatings. This is interesting for an enhanced corrosion protection performance. For example, $\mathrm{He}$ at al. combined $\mathrm{Al}$ with $\mathrm{Zn}$ for an enhanced corrosion resistance of carbon steel [6]. Baldwin et al. fabricated an alloy of $\mathrm{Al}$ and $\mathrm{Mg}$ for coating steel [7]. Creus et al. combined $\mathrm{Al}$ and $\mathrm{Cr}$ and magnetron sputtered an amorphous coating onto mild steel for corrosion protection [8]. Another approach by the same group is the combination of $\mathrm{Al}$ and $\mathrm{Ti}$ in cathodic arc PVD for steel in a multilayer method where the columnar structure of $\mathrm{Al}$ is disrupted by the Ti layer, thus leading to better corrosion resistance [9]. In the present set-up, one could either use an alloy sputter target, or a segmented sputter target consisting of different metals for forming an alloy coating. For a multilayer coating, however, a change in sputter target would be required. In earlier work, similar techniques have been used to coat other three-dimensional objects, including the inner walls of cylinders and tubes $[10,11,12]$. In this case, the sputter target, either a cone or a rotating ellipse, had been introduced into the hollow sample, giving uniform coatings for corrosion or wear protection.

These examples show that ion beam techniques have the potential for treating non-flat samples successfully.

\section{SUMMARY}

It has been demonstrated that ion beam sputter coating is a reasonable alternative to other PVD coating methods, even for three-dimensional objects, such as rings, if a designated sputter target with a substrate-adapted form is used. Coating adhesion and electrochemical corrosion measurements showed that a very good corrosion protection performance over the whole ring sample can be obtained. Apparently, an enhanced substrate temperature is not required, in contrast to sputter coating techniques which usually do not give dense coating structures at low substrate temperature.

\section{REFERENCES}

[1] J.J. Cuomo, S.M. Rossnagel, H.R. Kaufman (Eds.), "Handbook of Ion Beam Processing Technology", Noyes Publication, Park Ridge, NJ (1989)

[2] J.A. Thornton, J.E. Greene, "Ion Beam Sputtering", in: R.F. Bunshah (Ed.), "Handbook of Deposition Technologies for Films and Coatings", Noyes Publication, Park Ridge, NJ (1994), p. 327

[3] W. Ensinger, "Protection against aqueous corrosion by ion implantation and ion beam assisted deposition", in: Y. Pauleau, P.B. Barna (Eds.), "Protective Coatings and Thin Films: Synthesis, Characterization and Applications", Kluwer Academic Publishers, Dordrecht (1997) p. 585

[4] J.F. Ziegler, J.P. Biersack, and U. Littmark, "Transport of Ions in Matter", Pergamon Press, New York (1996)

[5] J.F. Ziegler, Nucl. Instr. Meth. Phys. Res. B 219-220, 1027-1036 (2004): Software package SRIM-2012.03

[6] Y. He, D. Li, D. Wang, Z. Zhang, H. Qi, W. Gao, Materials Letters 56 (4), 554-559 (2002)

[7] K.R. Baldwin, R.I. Bates, R.D. Arnell, C.J.E. Smith, Corrosion Science 38 (1), 155-170 (1996)

[8] J. Creus, A. Billard, F. Sanchette, Thin Solid Films 446 (1-2), 1-9 (2004)

[9] J. Creus, H. Idrissi, H. Mazille, F. Sanchette, P. Jacquot, Thin Solid Films 346 (1-2) 150-154 (1999)

[10] O. Lensch, Th. Kraus, Ch. Sundermann, B. Enders, W. Ensinger, Surf. Coat. Technol. 158-159, 599-603 (2002)

[11] W. Ensinger, Surf. Coat. Technol. 177-178, 264-270 (2004)

[12] W. Ensinger, B. Enders, J. v. Ringleben, R. Hatada, K. Baba, Trans. Mat. Res. Soc. Japan 35, 751-754 (2010)

(Received October 21, 2012; Accepted December 27, 2012) 